\title{
Improving Quality of Service for Mobility Aware of Multi-path Routing Protocol for Load Balancing in Mobile Ad-Hoc Network
}

\author{
V. Manju ${ }^{1}$ and R. Vadivel $^{2}$ \\ ${ }^{1}$ Research Scholar and ${ }^{2}$ Assistant Professor \\ Department of Information Technology, Bharathiar University, Coimbatore, Tamil Nadu, India \\ Email:manjuajura@gmail.com,vlr_vadivel@yahoo.co.in
}

\begin{abstract}
In Mobile Ad hoc Network (MANET), the single path QoS routing is disadvantageous as it may cause interference, fading, collision and link failures. For QoS routing, the load balancing is necessary, because it allows a router to take advantage of best of multiple shortest paths to a given destination. It also avoids congestion within the network using multipath. While using the MQRLB, the Load balancing may cause some of the major issues such as Link failure, Out of range, Limited Bandwidth, due to this the load balancing in multipath function is not efficient.To overcome these issues, in this paper, MMQRLBMultipath Mobility Aware QoS Routing Load Balancingwith AODV(Ad-hoc on demand distance vector)protocol is proposed to improve the $\mathrm{QoS}$ in Load balancing.

Keywords: MANET, Multipath, MMQRLB, QoS, Load balancing
\end{abstract}

\section{I.INTRODUCTION}

The Wireless ad-hoc networks are collections of wireless nodes in whichit communicate directly over a common wireless channel. In MANET, the nodes are connected over radio waves. The nodes are equipped with wireless transceiver[1].The importance of computers in our daily life increases and it also sets new demands for connectivity. The Wired solutions are available for a long time but there is increasing demand on functioning wireless solutions for connecting to the internet,sending and reading E-mail messages, changing information in a meeting and so on. In Latin ad-hoc means "for this", further meaning "for this purpose only" [2].It is a good and symbolic description of the design why ad hoc networks are needed.In network the wireless Mobile ad hoc network can be set up anywhere without any need for external infrastructure like wires or base stations.MANET is an infrastructure less network consists of self-configuring mobile devices which can move independently in any direction and leads to frequent modification in the transmission links with respect to other devices.

The Mobile Ad-hoc Network (MANET) is a collection of mobile nodes in which it communicate with each other via wireless links either directly or relying on other nodes as routers [3].The main harms in ad hoc networks are routing and characteristic of wireless communication. In infrastructure networks a node can communicate with all nodes within the same cell.Some vital characteristics of mobile ad-hoc networks arepeer-to-peer fashion during data transfer, dynamic topologyand mobility of nodes and in real-time such networks are heterogeneous.

The Routing protocols in ad-hoc wireless networks can be classified into three broad categories. This classification is based on the routing information update mechanism. They are Proactive /Table driven protocols, Reactive / Ondemand protocols, and Hybrid routing protocols in proactive routing protocols the routes to all the destination nodes are determined at their establishment and maintained by using a periodic route update process. The proactive routing protocols are WRP, GSR, FSR, STAR, DSDV, CGSR, HSR, OLSR .In reactive protocols, the routes are determined when they are required by the source using a route discovery process. The reactive routing protocols are DSR,AODV, LMR, TORA, ABR, and SSA. Hybrid routing protocols combines the properties of the Proactive and Reactive protocols into one. Hybrid routing protocols are ZRP, DST, DDR[4].

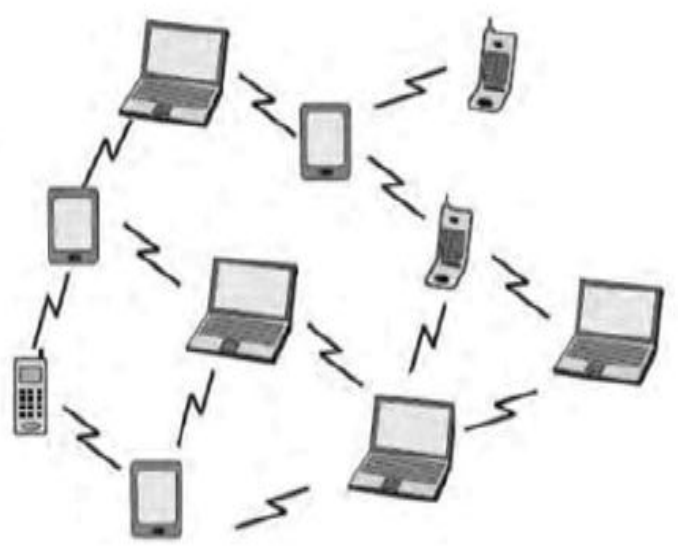

Fig. 1 Mobile Ad hoc Network

The mobile ad-hoc wireless network is a collection of more than one devices or nodes or terminals by means ofwireless communications and networking capability that communicate with each other without the assist of any central administrator and also the wireless nodes that can dynamically exchange information without using any existing fixed network infrastructure.

\section{A. Load Balanced Routing}

The main goal through load balancing is to make more use of available network resources in order to minimize the risk 
of traffic congestion [5]. Load balancing is a technique in which the existing multi paths will forward the packets from mobile nodes which have enough capacity is called Load balancing [6]. The overall network throughput can be increased and a better QoS can be provided for the network due to load balancinghelps to reduce the congestion during packet transmission [7].

\section{B. Multi-path Routing}

The Multipath Routing is the handful of traffic from a source node to a destination node over multiple paths through the network [8].The Multi-path routing allows transferring nodes in to multiple paths between a single source and single destination node during a single route discovery. The mainprincipal issuesin MANET such as scalability, security, network lifetime, etc. can be handled by the multi-path routing protocols [9]. The Multipath routing protocols are better than unipath protocols, because if there is a link breakage there could be another path to transmit the packets. Probability of link breakage is more because of the dynamic topology of nodes in MANET[10]. Therefore it is very difficult to maintain QoS.

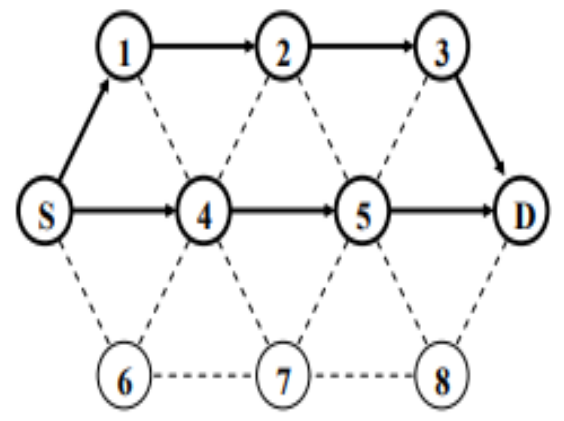

Fig. 2 Multipath Routing Protocol

\section{C.Ad-hoc on demand Distance Vector}

AODV stands for Ad-hoc On demand Distance Vector. AODV is an On Demand Routing Protocol [11]. Since it is a reactive protocol the routes are determined only when it is needed.Whenever an AODV nodeor router receives a request to send a communication, it checks it's Routing Table for route existence.Each Routing Table entry consists ofNext Hop Address, Hop Count Destination Address [12]. The AODVnodesusetwo typesof messages to communicate among each other they are Routediscovery, RouteMaintenance. The Route Request (RREQ) and the Route Reply (RREP) messages are used for route discovery. Route Error (RERR) messages and HELLO messages are used for route maintenance [13].

\section{LITERATURE REVIEW}

Mobile Ad-Hoc Network (MANET) has become more popular in recent years because of its features like mobility and deployed nature of the networks in MANET. In mobile ad hoc network, it finds the shortest path to transmit the packet from source to destination using Multipath [14]. Since load balancing has some of the major issues like link failure, out of range during transmission from source to destination.

S.Venkatasubramanianan and Dr.N.P.Goplan [5],the QoS based ROBUST Multi-path Routing (QRMR)protocol for mobile ad hoc networks wasdeveloped.The mobilead-hoc networks to allot weights to, depending on the metrics link quality, individuallinkschannel quality and end-to-end delay. The individual link weights are mutual into a routing metric to validate the load balancing and interference between links using the same channel.

Dr. B.S Pradeep[8], The Ad Hoc On-Demand Distance Vector AODV) routing protocol is one of the well-known and efficient on-demand MANET protocols. AODV protocol does not support Quality of Service (QoS) and also has no load balancing mechanism. Now a days,variousimprovements has made to the AODV protocol to present QoS and load balancing features. This is done by accumulating two extensions to the messages used whilediscoveringroute. The packet layer simulation model with physical layerand media access control (MAC) models is used to study the performance of both QoS-AODV protocols and AODV.

S.Venkatasubramanianan and Dr.N.P.Goplan [7], single path QoS routing is disadvantageous since it may cause fading, interference, and collision and link failures. For QoS routing, load balancing is essential since it allows a router to take advantage of best multiple paths to a given destination. It can minimize the maximum utilization while supporting the same traffic demands. It reacts quickly to changes in traffic demands, link failures, and traffic spikes. It also avoids congestion within the network. Therefore, considering the advantages of load balancing, a multi-path routing for load balancing (MQRLB) is proposed in this paper.

Initially, Route Discovery is initiated when the source node attempts to discover disjoint routes to the destination. After multiple disjoint routes are established, the balancing function and the forwarding function for each path is calculated, based on which the load unbalance condition is checked. In case of load unbalance, load distribution is done by adding redundant codes along with the data and transmitting through multiple paths. Thus QoS routing can be processed through multiple successful paths using load balancing.

\section{PROBLEM IDENTIFICATION}

In this paper [5], a mobility aware QoS multipath routing protocol for MANET is proposed. In this techniques Mobility aware is performed to create a quality of load balancing in multipath. The load balancing has some of the major issues in multipath routing protocol, end to end delay, packet dropping, imbalanced energy consumption and inefficiency.To overcome these issues, Mobility aware is proposed to avoid link failure, out of range, delay. Here, the mobility parameters help to predict when the neighbor node 
is out of range during packet transmission and avoids the packet loss during transmission. Therefore it providesQos Load balancing in multipath routing protocol.

\section{PERFORMANCE METRICS}

1. Average Packet Delivery Ratio: It is the number of packets received successfully and the total number of packets transmitted.

2. Average end-to-end delay: The average end-to-enddelay is averaged over all enduring data packets from the sources to the destinations.

3. Control overhead: The control overhead is well demarcated as the total number of routing control packets normalized by the total number of received data packets.

4. Throughput: It is the number of packets successfully received by the receiver.

\section{V.RESULT AND DISCUSSION}

In order to evaluate the performance of the proposed mechanism, Network Simulator 2 (ns-2) is used.

\section{A. Simulation Configuration}

The Simulation is carried out using the Network simulator 2 (NS-2) as shown in the Table 1 below.

TABLE I SIMULATION PARAMETERS

\begin{tabular}{|l|l|}
\hline Platform & Ubuntu \\
\hline Ns Version & Ns 2.34 \\
\hline No. of Nodes & 50 \\
\hline Area & $1000 * 1000 \mathrm{~m}$ \\
\hline MAC & 802.11 \\
\hline Range & $250 \mathrm{~m}$ \\
\hline Simulation time & $100 \mathrm{sec}$ \\
\hline Traffic source & CBR \\
\hline Rate & Kbps \\
\hline Packet size & 512 bytes \\
\hline Protocol & AODV \\
\hline Speed & 5 to 20 \\
\hline Pause time & $5,10,15,20$ \\
\hline
\end{tabular}

In Table I, These parameters are used to provide a Network performance using Ns2 Simulator .i.e. No. of Nodes use to transmit a packet and area size of the network simulator. Here, The AODV protocol used for multipath transmission.

\section{B. Based on PauseTime}

In Firstexperiment, measures the performance of the protocol by varying the no. of nodes as 15,25 and 50 .

In Fig. 3, shows the pause time of Average Throughput are high when compared with the existing MQRLB. The throughput is higher than MQRLB. This helps to avoid dropping the packets while transmission.

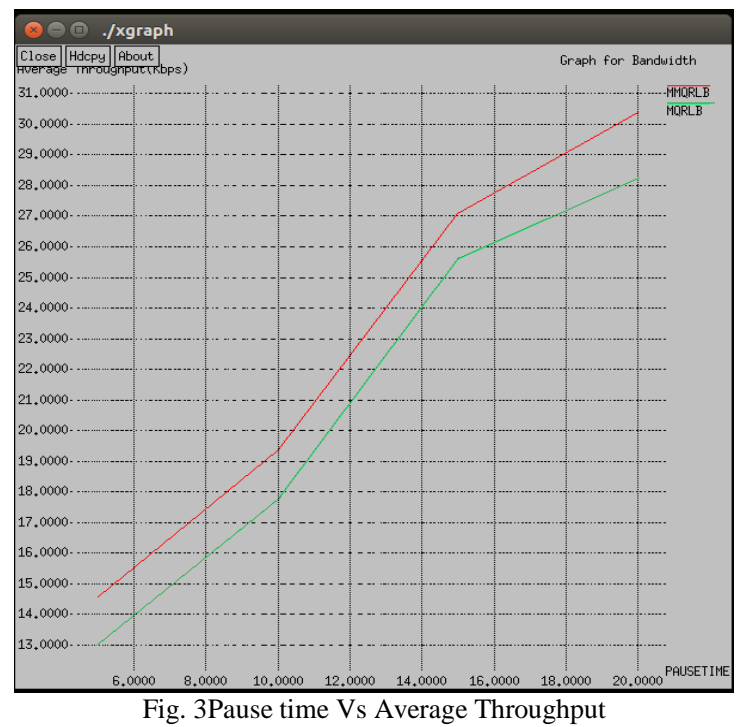

In Fig. 4, shows the pause time of Packet Delivery Ratio are higher when compared with the existing MQRLB. MMQRLB has received $62 \%$ more packets when compared to MQRLB.

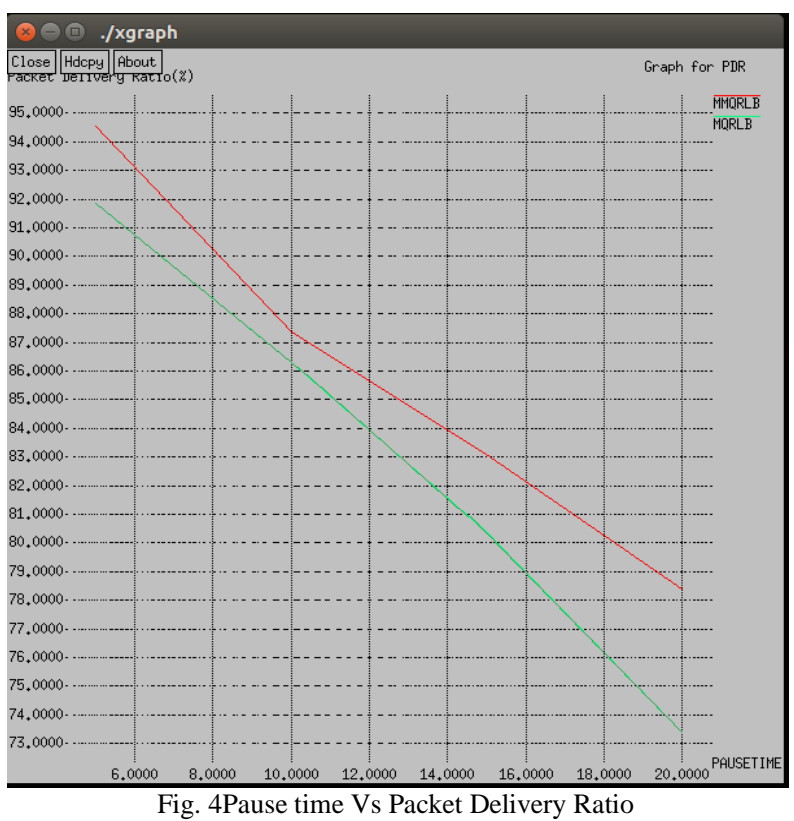

Fig. 5 shows the pause time of Average Delay of MMQRLB is less when compared with the existing MQRLB Average delay. The delay of a network specifies how long does it takes for a bit of data to travel across the network from one node to another. Moreover, it consumes lesser along the routes. Ref Fig.5

In Fig. 6, the pause time of Control Overhead is less when compared with MMQRLB. Here, the performance of the protocols by varying the no. of nodes as 15, 25 and 50. Ref Fig.6. 


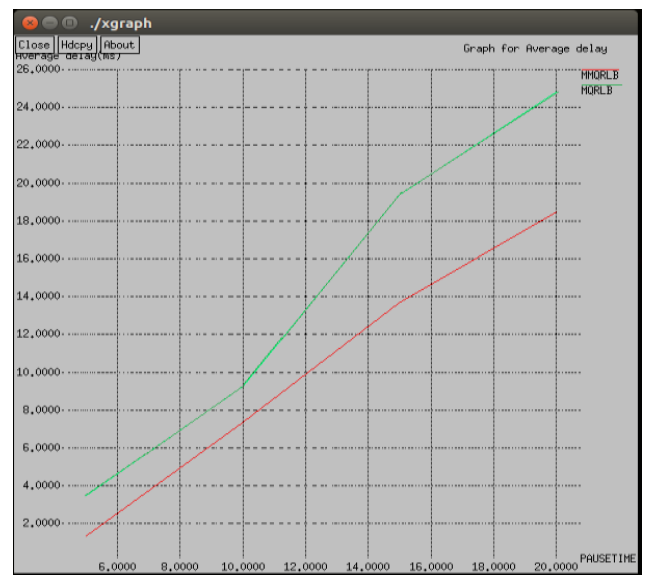

Fig. 5 Pause Time Vs. Average delay

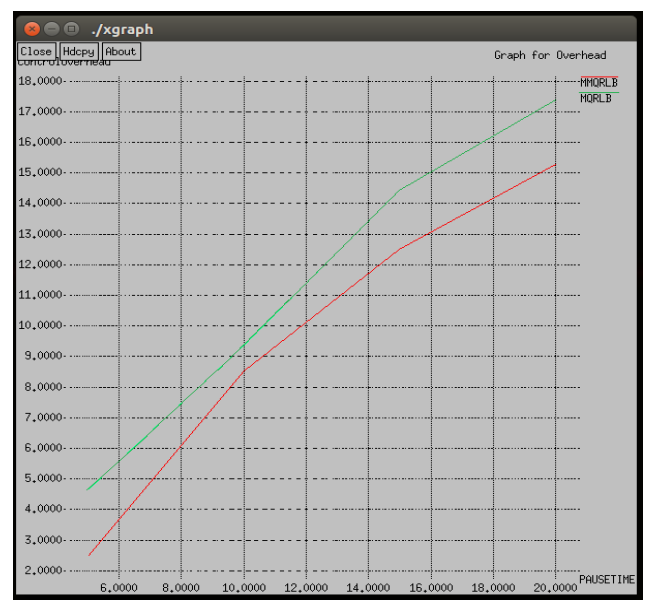

Fig. 6 Pause time Vs. Control Overhead

\section{Based on Speed}

In Second experiment, analyzing the metrics by varying the speed as $5,10,15$, and $20 \mathrm{~m} / \mathrm{s}$. The below $\mathrm{x}$ graph is given based on speed.

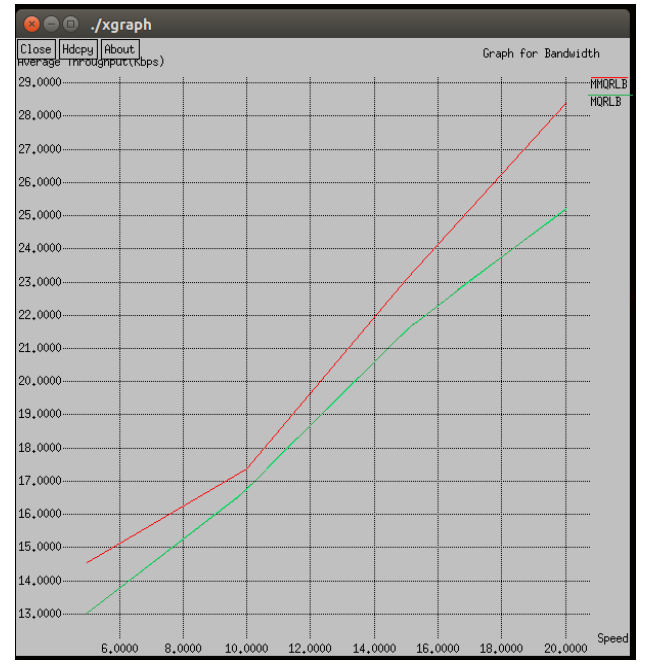

Fig. 7 Speed Vs. Average Throughput

The received Average Throughput for the proposed is higher than the existing MQRLB.

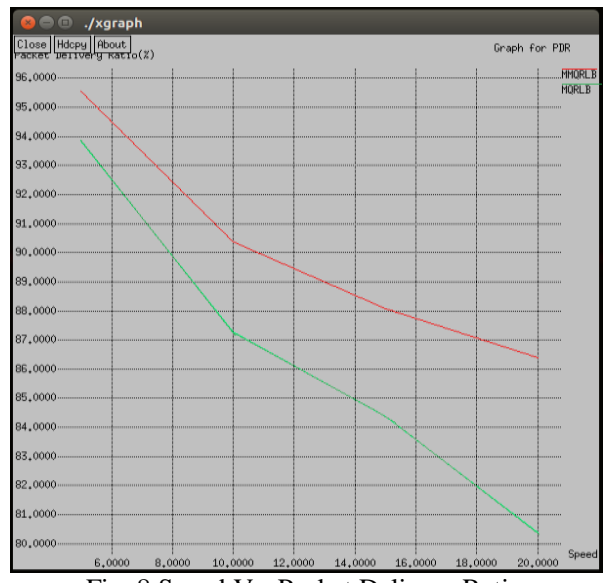

Fig. 8 Speed Vs. Packet Delivery Ratio

The received Average Throughput for the proposed is higher than the existing MQRLB.

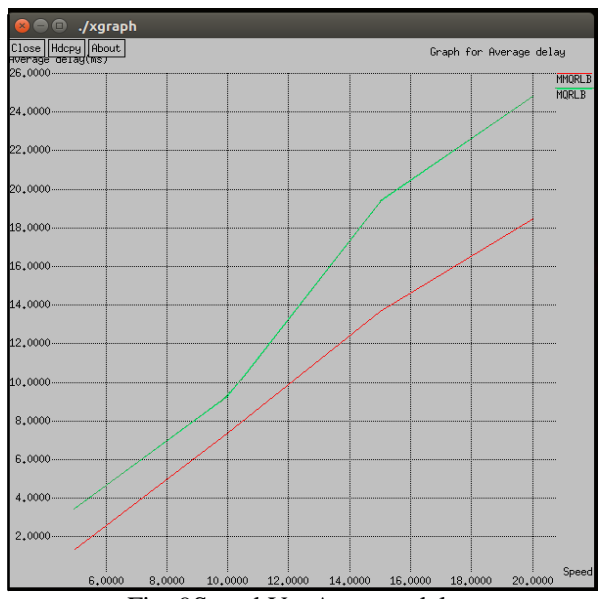

Fig. 9Speed Vs. Average delay

The received Packet Delivery Ratio for the proposed is higher than the existing MQRLB.

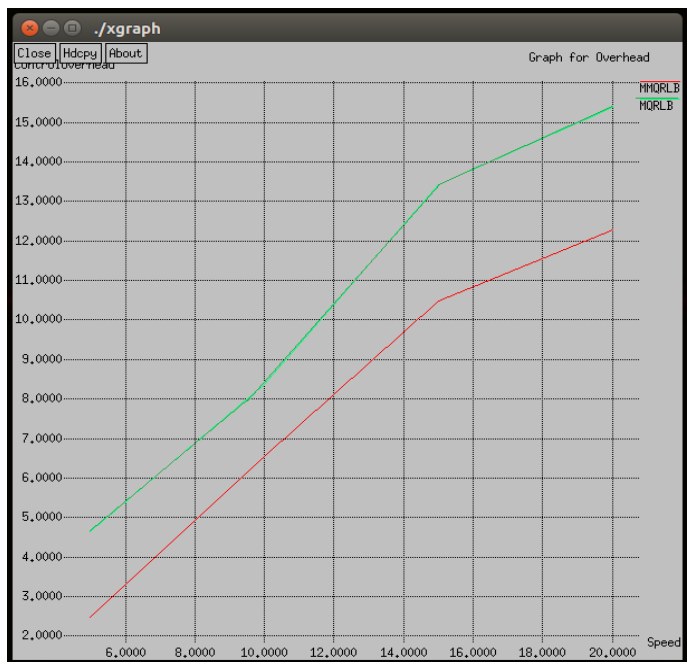

Fig. 10Speed Vs. Control Overhead

The received for Average delays the proposed is less than the existing MQRLB.The received Control Overhead for the proposed is less than the existing MQRLB. 


\section{CONCLUSION AND FUTURE WORK}

In MANET, load balancing technique plays animportant role in order to achieve the QoS solutions. Load balancing is one of the key areas pertaining to research in the field of mobile ad-hoc networks. The conventional MANET routing protocols suffering from more routing overhead and decreased packet delivery ratio due to non-addressing of the load balance in MANET communications.In this paper, mobility aware routing protocols have been discussed. These protocols use several QoS metrics like delay, bandwidth, routing overhead. The proposed MMQRLB helps to resolve the existing MQRLB drawbacks. In future, traffic splitting can be implemented in this work to get better QoS in Multipath load balancing in MANET.

\section{REFERENCES}

[1] Dr.S.S.Dhenakaran and A. Parvathavarthini, "An Overview of Routing Protocols in Mobile Ad-Hoc Network," International Journal of Advanced Research in Computer Science and Software Engineering, Vol.3, Issue 2, February 2013.

[2] S. Murthy and J. J. Garcia-Luna-Aceves, "An efficient routing protocol for wireless networks," Mobile Networks and Applications, Vol. 1, no. 2, pp. 183-197, 1996.

[3] Pai-Hsiang Hsiao, Adon Hwang, H. T. Kung, and Dario Vlah, "LoadBalancing Routing for Wireless Access Networks", Proc. IEEE Infocom, pp. 986-995, 2001.

[4] Abdulaziz Al- Nahari and Mohd MurtadhaMohamad, "ReceiverBased Ad Hoc On Demand Multipath Routing Protocol for Mobile Ad Hoc Networks", PLOSONE |DOI:10.1371/journal. Pone.0156670 , June 3, 2016.
[5] S.Venkatasubramanian and N.P.Gopalan,"Multi-path QoS Routing Protocol for Load Balancing in MANET",International Journal of Networking \& Parallel Computing, Vol. 1, Issue 3, Dec2012Jan2013.

[6] D. Maheshwari and R. Nedunchezhian, "Load Balancing in Mobile Ad Hoc Networks: A Survey", International Journal of Computer Applications, Vol.59- No.16, December 2012.

[7] Chengyong Liu, Kezhong Liu and Layuan Li, "Research of QoSaware Routing Protocol with Load Balancing for Mobile Ad hoc Networks", IEEE,2008.

[8] S.Venkatasubramaniananand Dr.N.P.Gopalan,"A QoS-Based Robust Multipath Routing Protocol for Mobile Adhoc Networks", IACSIT International Journal of Engineering and Technology, Vol.1, No.5, December, 2009.

[9] Stephen Mueller, Rose P. Tsang and Dipak Ghosal, "Multipath Routing in ad hoc networks: Issues and Challenges", pp.209234.Springer-Verlag Berlin Heidelberg, 2004.

[10] Dr. B.S Pradeep and Mrs. S.Soumya, "A New Method for Load Balancing and QOS in On demand Protocols-In the MANET's Perspective", International Journal of Advanced Networking and Applications, Vol.01, Issue: 04, Pages: 275-28, 2010.

[11] Neerja Khatri and Arvind Kumar, "Analysing Performance Of AODV In MANET: A SURVEY", International Journal of Scientific and Engineering Research, Vol.3, Issue 6, June 2012.

[12] Ipsita Panda, "A Survey on Routing Protocols of MANETs by Using QoS Metrics", International Journal of Advanced Research in Computer Science and Software Engineering, Vol.2, Issue 10, October 2012.

[13] RoopaliGargand Guneet Kaur, "Survey on Multipath QoS Routing Protocols in MANET", International Journal of Advanced Research in Electrical, Electronics and Instrumentation Engineering, Vol. 3, Issue 1, January 2014.

[14] Karunya Rathan and S.Emalda Roslin, "A Survey on Routing Protocols and Load Balancing Techniques in Wireless Mesh Networks",International Conference on Intelligent Computing and Control, 2017. 